\begin{tabular}{c} 
International Journal of Advanced Astronomy, 2(2) (2014) 27-28 \\
International Journal of Advanced Astronomy \\
Journal home page: $\begin{array}{c}\text { www.sciencepubco.com/index.php/IJAA } \\
\text { doi: } 10.14419 / \text { ija. } 2 \text { 2i } 2.3088 \\
\text { Research Paper }\end{array}$ \\
\hline
\end{tabular}

\title{
An appraisal of Milgrom's fitting formula by the antigraviton-graviton theory
}

\author{
Wing-Hong Wong (黃穎航) *,Wing-To Wong (黃穎濤),Wing-Keung Wong (黃穎強), Ling-Mei Wong (黃靈美) \\ China Evangelical Seminary, Taipei \\ *Corresponding author E-mail: jwhwong@ces.org.tw
}

\begin{abstract}
On one hand, the Wong's antigraviton-graviton theory (AGT), which may also be called a new quantum gravity theory, predicts that at the limit of infinite radius, the effective acceleration is proportional to the square root of the Newtonian gravitational acceleration. Thus it partially supports Milgrom's fitting formula (MIFF). On the other hand, our AGT discovers that the definition of the critical acceleration as a universal parameter by MIFF is not adequately valid.
\end{abstract}

Keywords: Cosmology: Theory, Dark Matter, Galaxies: Kinematics And Dynamics, Gravitation, Large-Scale Structure Of Universe.

\section{Introduction}

According to Newton's second law of motion, $F=m a$ where $F$ is the force acting on a mass $m$, and $a$ is the acceleration produced. In 1983, the first alternative model to the dark matter hypothesis, called Modified Newtonian Dynamics (MOND), was proposed by Milgrom, who suggested an ad hoc modification of the second law: when the acceleration is much smaller than a universal critical acceleration $a_{\mathrm{o}}$, force becomes proportional to the square of acceleration; but if the acceleration is much larger than $a_{\mathrm{o}}$, force is proportional to acceleration as usual. Milgrom emphasized that MOND "does not constitute a theory", and can at most be interpreted as an "effective working formula", which has "limited applicability" [1]. Astronomical data of galaxies, galactic clusters and superclusters have given considerable empirical support to Milgrom's fitting formula (MIFF). In the following, we shall derive an acceleration formula in the limit of infinite distance, according to the Wong's antigraviton-graviton theory (AGT) [2], which may also be called a new quantum gravity theory, and compare it with MIFF.

\section{Theory}

According to our AGT [2], when the radius R is less than or equal to the gravitational scale-length $R_{\mathrm{o}}$, Newtonian gravity theory (NGT) is correct. Thus the centripetal acceleration of a star which is at a radius of $R_{\mathrm{o}}$ from the galactic center is

$$
a_{\mathrm{o}}\left(R_{\mathrm{o}}\right)=G_{\mathrm{n}} M\left(R_{\mathrm{o}}\right) / R_{\mathrm{o}}^{2}
$$

where $M\left(R_{\mathrm{o}}\right)$ is the central mass at the radius $R_{\mathrm{o}}$. According to NGT, the centripetal gravitational acceleration at any radius $\mathrm{R}$ is

$a_{\mathrm{n}}(R)=G_{\mathrm{n}} M(R) / R^{2}$, where $M(R)$ denotes the central mass at the radius $\mathrm{R}$. In reference [2], it has been shown that the effect of the cooperation of antigravitons with gravitons is that the central mass $M(R)$ has been amplified by an amplification factor of

$\mu(R)=\cosh [R / \lambda(R)] / \cosh (1)$.

Hence, according to our antigraviton-graviton theory (AGT), using eq. (3), the centripetal acceleration at radius $\mathrm{R}$ is

$a_{\mathrm{q}}(R)=\frac{G_{\mathrm{n}} M(R) \times \mu(R)}{R^{2}}$.

In reference [2], it has also been shown that the distance dependence of the expectation value of the graviton wavelength is

$\lambda_{\mathrm{A}}(R)=R_{\mathrm{o}}\left[1+\frac{\left(R / R_{\mathrm{o}}\right)-1}{1+\ln \left(R / R_{\mathrm{o}}\right)}\right]$.

From eq. (5), it can be shown that

$R \rightarrow \infty, \lambda_{\mathrm{A}} \rightarrow \frac{R}{\ln \left(R / R_{\mathrm{o}}\right)}$

Eq. (6) yields

$R \rightarrow \infty, \frac{R}{\lambda_{\mathrm{A}}} \rightarrow \ln \left(R / R_{\mathrm{o}}\right)$

$R \rightarrow \infty, \cosh \left(R / \lambda_{\mathrm{A}}\right)=\cosh \left[\ln \left(R / R_{\mathrm{o}}\right)\right]=0.5\left(R / R_{\mathrm{o}}\right)$

Using eq. (3) and eq. (8), we obtain

$$
R \rightarrow \infty, \mu(R) \rightarrow \frac{R}{2 R_{\mathrm{o}} \cosh (1)}
$$


Substituting eq. (9) into eq. (4), we have

$$
R \rightarrow \infty, a_{\mathrm{q}}(R) \rightarrow \frac{G_{\mathrm{n}} M(R)}{2 \cosh (1) R_{\mathrm{o}} R} .
$$

From eq. (1), (2) and (10), we get

$$
R \rightarrow \infty, a_{\mathrm{q}}(R) \rightarrow \frac{\eta}{\sqrt{2 \cosh (1)}} \sqrt{a_{\mathrm{o}}\left(R_{\mathrm{o}}\right) a_{\mathrm{n}}(R)}=\frac{\eta \sqrt{a_{\mathrm{o}}\left(R_{\mathrm{o}}\right) a_{\mathrm{n}}(R)}}{1.756747},
$$

where $\eta$ is defined as the mass ratio

$$
\eta=M(\infty) / M\left(R_{\mathrm{o}}\right)
$$

In reference [2], we have shown that $R_{\mathrm{o}}$ is equal to $0.5 \pi$ times the radius of its radial centre of mass (RCM). In order to estimate $\eta$, let us take the approximation that the mass distribution of a galaxy (including both stars and gas) can be represented by an exponential function with a photometric scale-length $R_{\mathrm{ph}}$, it can be shown by integral calculus that the RCM is equal to $2 R_{\mathrm{ph}}$. Therefore,

$$
R_{\mathrm{o}}=\pi R_{\mathrm{ph}}
$$

Combining eq. (12) and (13), and using the formula for the calculation of mass for an optically thin model [3], it can be shown that

$$
M\left(R_{\mathrm{o}}\right) / M(\infty)=0.9567861 \text { (7 sig. fig.). }
$$

Thus the reciprocal $\eta$ is equal to 1.045166 ( 7 sig. fig.). Substituting this value of $\eta$ into eq. (11) we obtain

$$
R \rightarrow \infty, a_{\mathrm{q}}(R) \rightarrow 0.5949438 \sqrt{a_{\mathrm{o}}\left(R_{\mathrm{o}}\right) a_{\mathrm{n}}(R)} \approx 0.59 \sqrt{a_{\mathrm{o}}\left(R_{\mathrm{o}}\right) a_{\mathrm{n}}(R)}(2 \text { sig. fig.).(1 }
$$

Eq. (15) is calculated to 2 significant figures, as the above approximation, of using a single photometric scale-length $R_{\mathrm{ph}}$ in representing the galactic mass distribution, usually cannot attain accuracy better than about 3 per cent.

\section{Discussion}

From the above, our AGT found out that, for each spiral galaxy, when the radius reaches the gravitational scale-length $R_{\mathrm{o}}$, the acceleration attains a critical value $a_{\mathrm{o}}$, below which NGT is exact; but above which, NGT no longer applies. And $a_{\mathrm{o}}$ is found to be the centripetal acceleration of a star which is at a radius of $R_{\mathrm{o}}$ from the galactic center, i.e. $a_{\mathrm{o}}=a_{\mathrm{o}}\left(R_{\mathrm{o}}\right)$. Eq. (15) is very similar to Milgrom's fitting formula [MIFF], namely

$$
R \rightarrow \infty, a_{\mathrm{miff}}(R) \rightarrow \sqrt{a_{\mathrm{o}, \mathrm{miff}} a_{\mathrm{n}}(R)},
$$

where $a_{\text {miff }}(R) a_{\text {o,miff }}$ is the acceleration predicated by MIFF, and $a_{\mathrm{o} \text {,miff }}$ is the universal critical acceleration assumed by MIFF. Although eq. (15) predicts that at the limit of infinite radius, the effective acceleration is proportional to the square root of the Newtonian acceleration $a_{\mathrm{n}}(R)$ [1]; yet according to our AGT, the parameter $a_{\mathrm{o}}$ is defined as the acceleration at the gravitational scale-length $R_{\mathrm{o}}$. Consequently, the value of $a_{\mathrm{o}}$ depends on the mass distribution of the individual galaxy. This definition of $a_{\mathrm{o}}$ is different from MIFF, according to which $a_{\mathrm{o} \text {,miff }}$ is a universal constant. For a sample of nine spiral galaxies, Begeman, Broeils and Sanders found that the value of $a_{\mathrm{o} \text {,miff }}$ depends on each individual galaxy and the statistical average of $a_{\mathrm{o} \text {,miff }}$ is $1.21 \times 10^{-10} \mathrm{~m} \mathrm{~s}^{-2}$, with a dispersion of 0.27 (about 22\%) [4].

This relatively large dispersion suggests that the interpretation of our AGT is more accurate than that of Milgrom's definition of the critical acceleration ( $\left.a_{\mathrm{O} \text {,miff }}\right)$. Nevertheless, as much empirical support has been given to the ad hoc MIFF by galaxies, galactic clusters and superclusters, a similar support may be expected for our AGT, which is a gravitational theory derived from special relativity theory and quantum theory.

\section{Conclusion}

On one hand, since our AGT predicts that at the limit of infinite radius, the effective acceleration is proportional to the square root of the Newtonian acceleration $a_{\mathrm{n}}$, it partially supports MIFF. On the other hand, our AGT discovers that the definition of the critical acceleration ( $a_{\mathrm{O} \text {,miff }}$ ) as a universal constant by MIFF is not adequately valid.

\section{Acknowledgements}

We are grateful to the anonymous referees for their valuable comments. The encouragement from Tak-Hon Wong, Janice YuetChun Leung, and Vanessa Ue-Ching Wong is hereby gratefully acknowledged.

\section{References}

[1] Milgrom M (1983), A Modification of the Newtonian dynamics as a possible alternative to the hidden mass hypothesis. Astrophysical Journal, Part 1, Vol.270, 365-370.

[2] Wong W.H., Wong W.T., Wong W.K. \& Wong L.M., (2014), Discovery of the antigraviton verified by the rotation curve of NGC 6503. International Journal of Advanced Astronomy, Vol.2, No.1, 1-7

[3] Binney J \& Tremaine S (2008), Galactic Dynamics, 2nd edn. Princeton University Press, Princeton, NJ, p. 101.

[4] Begeman K.G., Broeils A.H. \& Sanders R.H., (1991), Extended rotation curves of spiral galaxies - Dark haloes and modified dynamics. Monthly Notices of the Royal Astronomical Society, Vol.249, 523-537. 